\title{
Characterizing the local charge density to determine complex magnetic correlations, and vice versa
}

\author{
N. Gauthier \\ Stanford University \& SLAC National Accelerator Laboratory, Stanford, California 94305, USA \\ nicolas.gauthier@stanford.edu
}

Crystal electric fields play an essential role in shaping the local electronic density of ions. For ions with strong spin-orbit coupling such as rare earths, this also results in defining the single-ion magnetic moment properties. Therefore, the study of crystal electric field levels is a common approach to establish the fundamental building blocks of the magnetic Hamiltonian. Indeed, the moment anisotropy and the single-ion wavefunction provide crucial information to describe complex magnetic materials such as spin liquids and multipolar systems [1,2]. In the first part of my presentation, I will illustrate this approach on the frustrated magnet $\mathrm{SrDy}_{2} \mathrm{O}_{4}$. This material exhibits two inequivalent zigzag chains of magnetic ions. The combination of low dimensionality and frustration inhibits long range order and only short-range magnetic correlations are observed down to $60 \mathrm{mK}$ [3]. Domain walls in the chains decay slowly and interchain interactions ultimately lead to their freezing, leading to a weakly fluctuating short range order [4]. The understanding of this complex behaviour could only be achieved from the knowledge of the moment anisotropies, established from the analysis of crystal field electric levels.

Above, we took advantage of strong spin-orbit coupling to determine magnetic properties by studying electric ones, i.e. the effect of crystal electric fields. This strong coupling between the electric charge and magnetic moment can also be used in the other direction: using magnetism to learn more about electric effects beyond the single-ion properties. As spin waves are collective excitations of the magnetic moments, the local charge densities can also sustain collective modes. Taking again the magnetic insulator $\mathrm{SrDy}_{2} \mathrm{O}_{4}$ as an example, I will demonstrate that neutron spectroscopy can measure these electric waves and that this observation is facilitated by the material's magnetism. Interestingly, our results indicate that electric interactions dominate the magnetic interactions in this case, although they remain hidden to most measurement techniques. This observation encourages a reassessment of the description of rareearth based magnets with unconventional properties.

[1] P. Santini, S. Carretta, G. Amoretti, R. Caciuffo, N. Magnani, G. H. Lander, Rev. Mod. Phys. 81, 807 (2009).

[2] R. Sibille, N. Gauthier, E. Lhotel, V. Porée, V. Pomjakushin, R. A. Ewings, T. G. Perring, J. Ollivier, A. Wildes, C. Ritter, T. C. Hansen, D. A. Keen, G. J. Nilsen, L. Keller, S. Petit \& T. Fennell, Nat. Phys. 16, 546 (2020).

[3] A. Fennell, V. Y. Pomjakushin, A. Uldry, B. Delley, B. Prévost, A. Désilets-Benoit, A. D. Bianchi, R. I. Bewley, B. R. Hansen, T. Klimczuk, R. J. Cava \& M. Kenzelmann, Phys. Rev. B 89, 224511 (2014).

[4] N. Gauthier, A. Fennell, B. Prévost, A.-C. Uldry, B. Delley, R. Sibille, A. Désilets-Benoit, H. A. Dabkowska, G. J. Nilsen, L.-P. Regnault, J. S. White, C. Niedermayer, V. Pomjakushin, A. D. Bianchi \& M. Kenzelmann, Phys. Rev. B 95, 134430 (2017).

Keywords: frustrated magnet; spin-orbit coupling; crystal electric field; electric interactions; collective modes 